\title{
FLUTUABILIDADE DO HOMEM MORTO NO CADINHO DO ALTO FORNO: UMA BREVE REVISÃO*
}

Kelly Cristina Ferreira ${ }^{1}$ Luiz Fernando Andrade Castro ${ }^{2}$

\section{Resumo}

$\mathrm{Na}$ operação do alto-forno é comum observar flutuações em seu comportamento, seguindo o ciclo de vazamento de gusa e escória. O acumulo de liquido no cadinho e sua influência no aumento da força hidrostática são as principais causas dessa flutuação.O cadinho é uma parte crucial na operação do alto-forno por muitas razões. No entanto, por ser o local de acumulo de gusa e escória, este deve ter volume suficiente e facilitar uma drenagem sem distúrbios. Por isto, o estado do redutor/combustível, chamado "homem morto" desempenha uma importante função. Este trabalho apresenta uma breve revisão sobre os principais indicadores do estado interno do homem morto no cadinho do alto-forno.

Palavras-chave: Cadinho; Homem morto; Estado de flutuabilidade.

\section{FLOATING STATE OF DEAD MAN ON THE BLAST FURNACE HEARTH: A BRIEF REVIEW}

\section{Abstract}

In the operation of a furnace is quite common to observe fluctuations in their behavior, following the cycles of casting pig iron and slag. The accumulation of liquid in the hearth and its influence on the descent of the load are the main cause of these fluctuations. The hearth is a crucial part in the operation of the ironmaking blast furnace for several reasons. Moreover, since it is a store for metal and slag it should have sufficient volume and facilitate undisturbed drainage. For this, the state of the hearth coke mainly the core wich is called "dead man" perform an important role. This paper presents a brief review of the main indicators of the internal state of the dead man in the blast furnace hearth.

Keywords: Hearth; Dead man; Floating state.

1 Engenheira Metalurgista, Mestranda em Tecnologia Mineral, Escola de Engenharia, Universidade Federal de Minas Gerais, Belo Horizonte, MG, Brasil.

2 Engenheiro Metalurgista, Professor, DEMET - Escola de Engenharia, Universidade Federal de Minas Gerais, Belo Horizonte, MG, Brasil. 


\section{INTRODUÇÃO}

É importante saber o estado do homem morto, se este está sobrenadante ou não, porque esta posição tem uma grande influência na distribuição dos gases e líquidos na parte inferior do alto-forno, pois esta afeta a distribuição de temperatura, o revestimento refratário e a drenagem dos líquidos do cadinho. É importante saber o estado de flutuação do homem morto para ser capaz de tomar ações de controle apropriadas para promover ou prevenir a flutuação do homem morto e predizer e prevenir o consumo acelerado do revestimento refratário do cadinho do alto-forno. Um bom controle dessas condições é necessário para assegurar uma longa campanha do cadinho e também uma produtividade elevada.

Se o volume acumulado de líquido no cadinho for suficiente, a força hidrostática age no coque submergido no banho e então o homem morto pode se elevar do fundo do cadinho. Um homem morto em flutuação é mais comum em pequenos altos-fornos.

Existe um vasto número de diferentes medições que refletem as condições internas do cadinho. Então, o problema é como os operadores poderiam extrair informações essenciais por trás dos dados. As condições internas do cadinho, especialmente o estado de flutuação do homem morto, devem ser obtidas através de medições indiretas, utilizando uma gama de indicadores que são baseados em medições já existentes.

A mudança no padrão de escoamento é resultado da transição de um homem morto encostado no fundo do cadinho para o estado flutuante, ambos possuem efeitos benéficos e maléficos a operação do forno.

Um homem morto sobrenadando o banho geralmente melhora as condições de drenagem dos líquidos e previne uma obstrução do homem morto, mas em contraposição o aumento do fluxo de gás no cadinho aumenta a deterioração do revestimento refratário e a variação de composição química do gusa tende a aumentar.

\section{INDICADORES DO ESTADO INTERNO DO CADINHO NO ALTO-FORNO}

\section{1 "Slag Delay"}

O "slag delay" é o tempo durante o qual apenas metal é vazado, antes da interface entre o gusa e a escória alcançar o furo de corrida.

A mudança no nível de gusa durante o vazamento depende do volume de líquido acumulado, e também o volume que depende da área da seção transversal do cadinho, da porosidade do homem morto e do estado de flutuação do homem morto. A relação entre o "slag delay" e o estado do cadinho e homem morto não é simples, mas usualmente um "slag delay" curto indica uma área de seção transversal grande, maior porosidade do homem morto ou um homem morto sobrenadando o banho.

Saxén et al. [1] estudaram o estado de flutuação do homem morto em dois altos-fornos usando o "slag delay" e os resultados são apresentados abaixo:

$\Delta t_{\text {sd }}=\Delta t_{\text {tap }}\left(1-\frac{A \varepsilon \Delta z_{i r, \text { end }}}{\Delta t_{p l} \dot{V}_{i r, \text { in }}}\right)$ para um homem morto sentado.

Eq. 1 


\section{Onde}

$\begin{array}{ll}\varepsilon & \text { Porosidade do coque } \\ A & \text { Área da seção transversal do cadinho } \\ \dot{V}_{i r, i n}, \dot{V}_{s l, i n} & \text { Fluxo de gusa e escória no cadinho } \\ \Delta z_{i r, \text { end }}=z_{t h}-z_{i r, e n d} & \text { Gusa no final da corrida } \\ \Delta t_{t a p} & \text { Duração da corrida. } \\ \Delta t_{p l} & \text { Período entre corridas }\end{array}$

$\mathrm{E}$, $\Delta t_{s d}=\Delta t_{\text {tap }}\left(1-\frac{A \varepsilon \Delta z_{i r, \text { end }}+(1-\varepsilon) \Delta V_{c f}}{\Delta t_{p l} \dot{V}_{i r, \text { in }}}\right) \quad$ Para um homem morto sobrenadante $\quad$ Eq. 2

Onde $\Delta V_{c f}$ é o volume da região de coque livre. Este pode ser estimado aplicando um balanço de forças nas forças atuantes no homem morto e assumindo um homem morto sentado.

Plotando os resultados do modelo para o alto-forno A assumindo um homem morto sentado pode-se notar uma excelente adequação dos dados medidos e estimados.

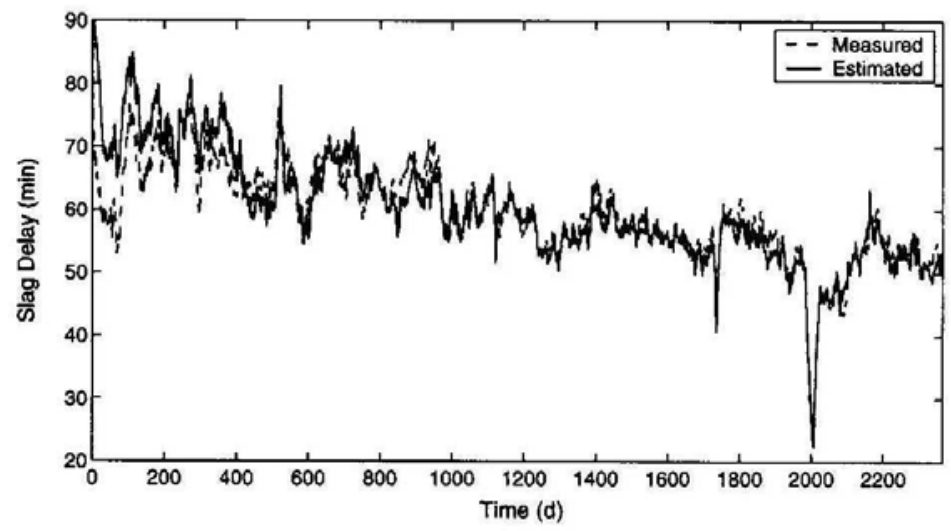

Figura 1 - "slag delay" observado e estimado para o alto-forno A assumindo um homem morto encostado no cadinho.

Plotando-se os resultados para o alto-forno B assumindo um homem morto sentado pode-se perceber a não-conformidade dos resultados.

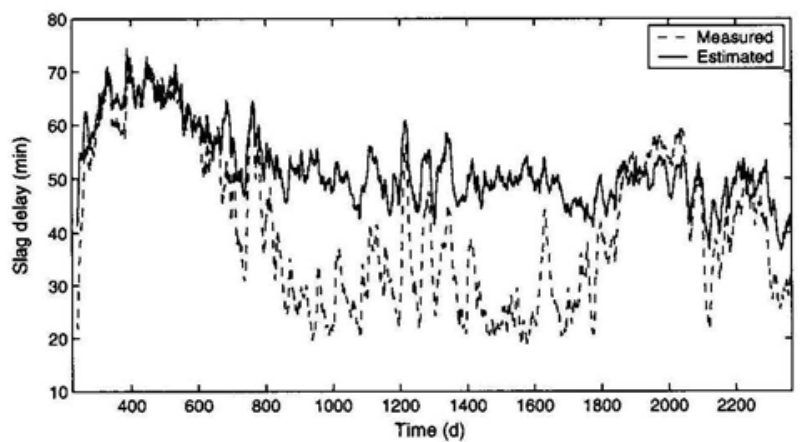

Figura 2 - "slag delay" observado e estimado no alto-forno B assumindo um homem morto encostado no cadinho. 
Então calculando o "slag delay" para um homem morto sobrenadando o banho para o alto-forno $B$ percebe-se a excelente adequação dos dados medidos e calculados.

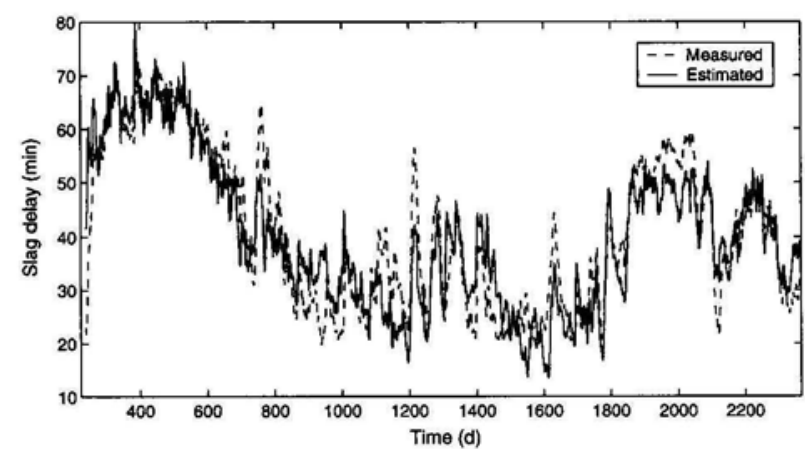

Figura 3 - "slag delay" observado e estimado no alto-forno B assumindo um homem morto sobrenadando o banho.

\section{2 Índice de Flutuação}

Para quantificar o efeito de um homem morto sobrenadando o banho, um índice é introduzido. $O$ índice de flutuação é definido como a mudança estimada no volume de coque livre entre o final da corrida anterior e o início da saída de escória, relativas à mudança no volume livre causado por alterações nos volumes de gusa e escória para o caso onde o homem morto está sobrenadando completamente no gusa.

$$
\Phi=\frac{\Delta V_{c f}}{\Delta V_{i r, s s}+\frac{\rho_{s l}}{\rho_{i r}} \Delta V_{s l, s s}}
$$

Onde $\Delta V_{s l, s s}$ é a mudança no volume da escória no início da corrida até o início do vazamento da escória.

\subsection{Composição Química do Gusa}

O gusa que está gotejamento no cadinho não está saturado em carbono, e devido à grande superfície de contato entre o gusa e a camada de coque ser maior que o contato entre o gusa e a camada refratária de carbono, a carburação acontece primariamente no homem morto, então se não há coque no fundo haverá menos gusa carburizado.

Torrkulla et al. [3] estudaram a correlação entre o "slag delay" e a carburação do gusa por um tempo maior que 600 dias.

Uma forte correlação entre as condições de drenagem na parte inferior do cadinho e a carburação está demonstrada no gráfico abaixo. Um "slag delay" curto implica em um homem morto sobrenadando o banho. 

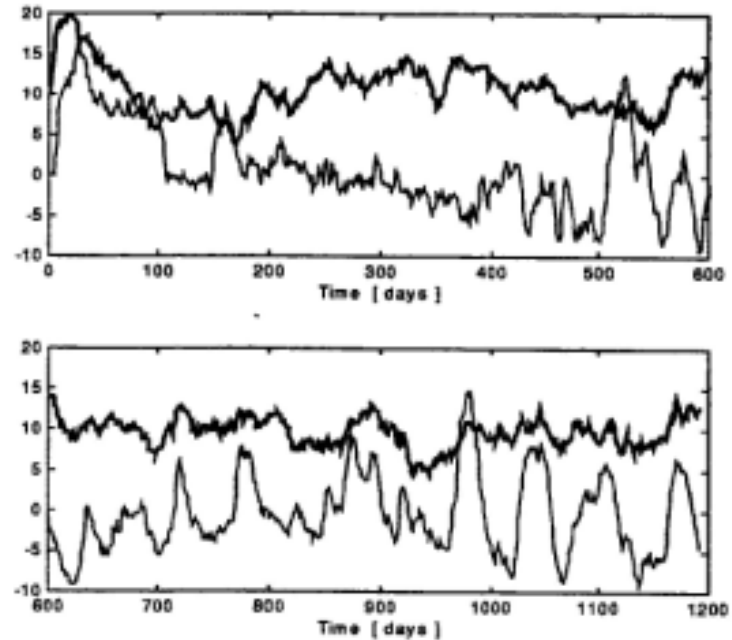

Figura 4 - O "slag delay" (linha normal) e o conteúdo de carbono (linha em negrito) do metal drenado [3].

\subsection{Força Eletromotriz (emf)}

Consiste de uma técnica para checar o nível de líquidos no cadinho através da implantação de eletrodos na carcaça do alto-forno e então é mensurada a diferença de potencial entre eletrodos normalmente localizados entre a posição das ventanteiras e abaixo do furo de corrida. Esta técnica não é muito acurada, sendo afetada por outros fatores como por exemplo a temperatura [4].

O gráfico abaixo mostra a relação entre o nível de líquidos e as medidas de emf.

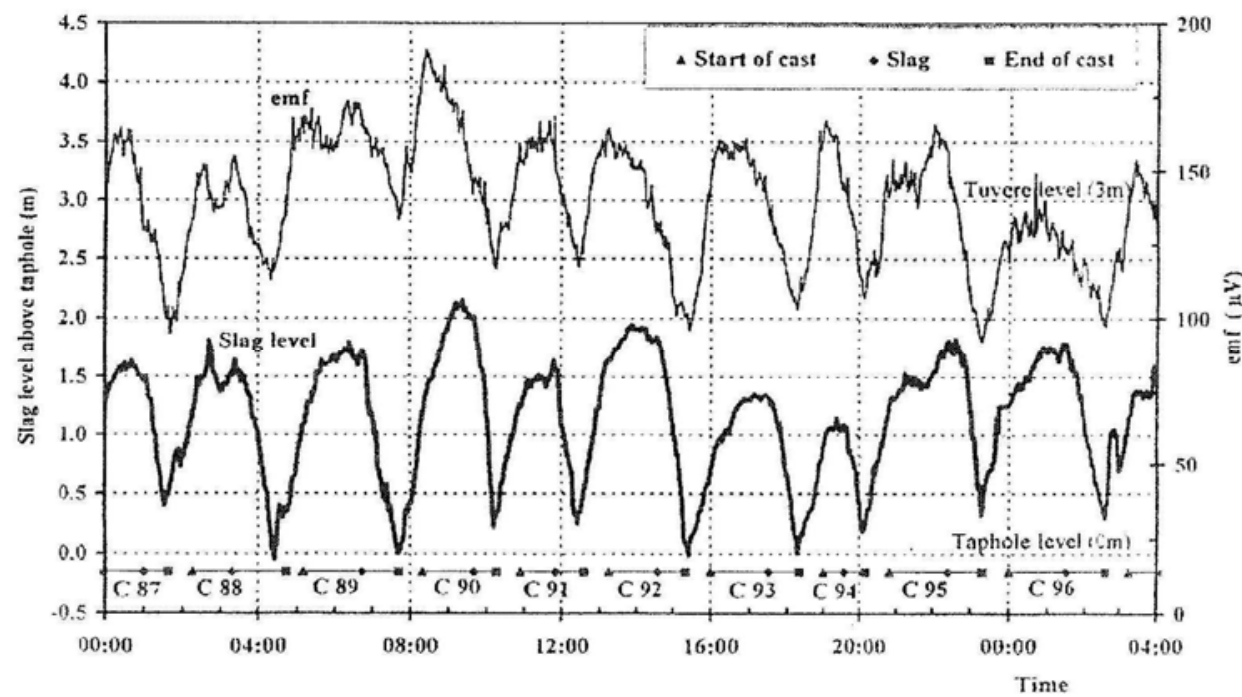

Figura 5 - Nível de escória e estimada com pressão estática e o sinal emf [2,6].

\subsection{Medições de Tensão}

Hattink et al. [5] desenvolveram um método para a tradução de medidas de tensão da carcaça do alto-forno em nível de líquidos no cadinho.

A tensão medida é a soma da pressão estática dos líquidos e da pressão dos gases e este tem sido um bom indicativo do nível de líquidos no cadinho.

Esta técnica pode ser útil para estimar o estado de flutuação do homem morto pois este influencia no nível de líquidos do cadinho do alto-forno. 


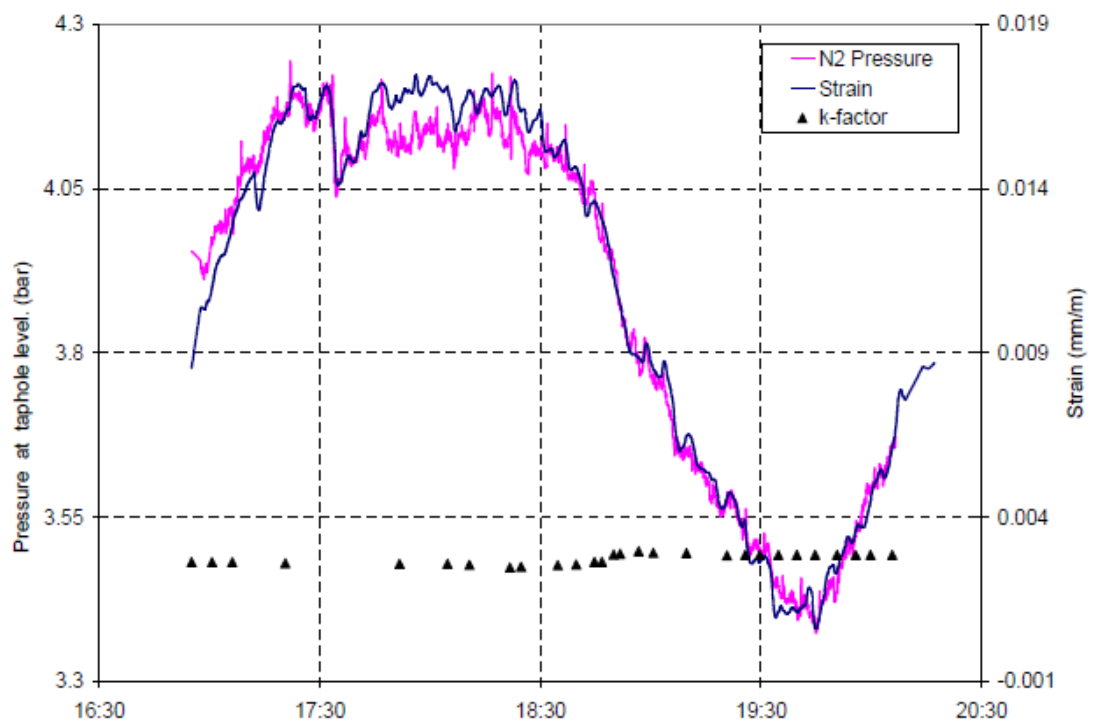

Figura 6 - Comparação entre a pressão e a tensão medida na carcaça do alto-forno ao nível do furo de corrida [5].

Na figura 6 é mostrada a pressão e a tensão medidas ao mesmo nível. As medidas de pressão são avaliadas através do uso de uma sonda. Ambas as medições apontam um resultado similar, indicando a possibilidade de medições de tensões serem indicativas da pressão interna do alto-forno.

Hatting [5] percebeu que há uma correlação linear entre a pressão estimada no cadinho e medições de tensão corrigidas porém ocorreu uma mudança na correlação entre uma campanha e outra.

Primeira campanha

Pressão Estimada $=\mathrm{k}_{0}+\mathrm{k}_{1} * \mathrm{SG}_{\text {taphole }}+\mathrm{k}_{2} * \mathrm{SG}_{\text {bottom }}$

Eq. 4

Segunda campanha

Pressão estimada $=\mathrm{k}_{0}+\mathrm{k}_{1}{ }^{*} \mathrm{SG}_{\text {taphole }}+\mathrm{k}_{2}{ }^{*} \mathrm{SG}_{\text {bottom }}+\mathrm{k}_{3}{ }^{*} \mathrm{~T}_{\text {shell }}$

Eq. 5

Com:

SGtaphole é a tensão medida ao nível do furo de corrida

SGbottom é a tensão medida ao nível do fundo do cadinho.

Tshell é a temperatura medida na carcaça do alto-forno.

$\mathrm{K}_{0}, \mathrm{k}_{1}$, k2, e k3 são parâmetros determinados pela regressão linear.

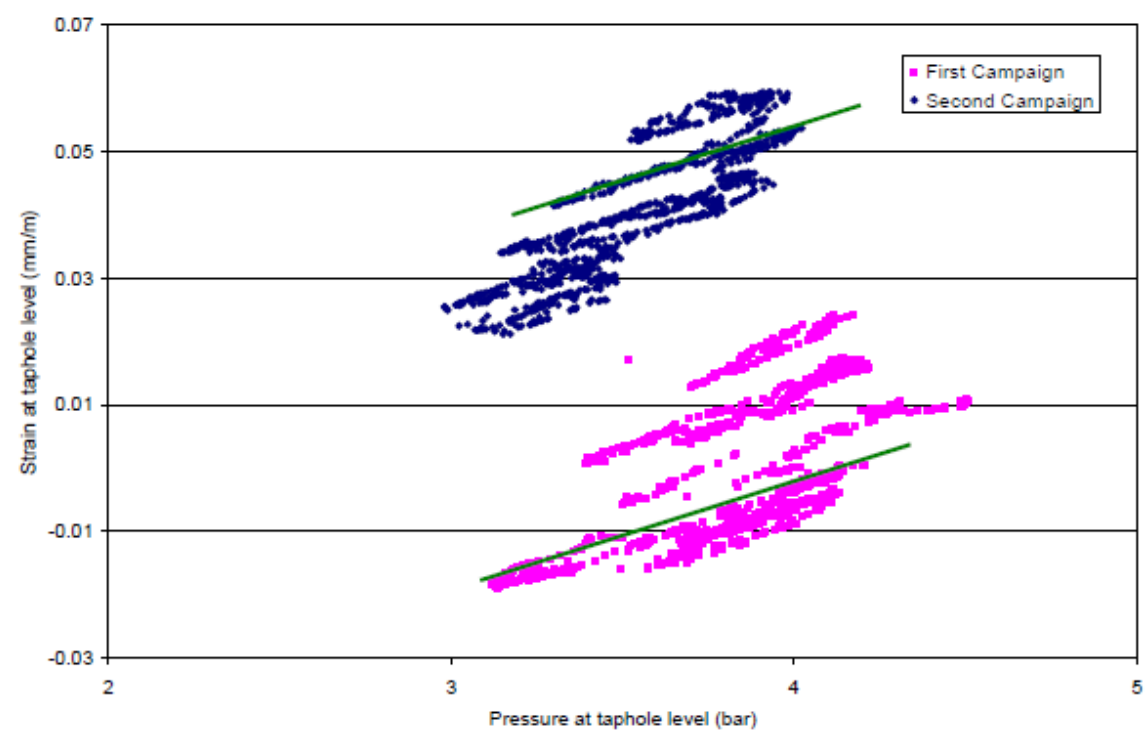

Figura 7 - Comparação entre a primeira e a segunda campanha. 
Devido a não observação de uma boa estimativa para as campanhas, foi encontrada uma fórmula que se adequasse melhor a ambas.

Pressão Estimada $=\mathrm{k}_{0}+\mathrm{k}_{1}{ }^{*} \mathrm{SG}_{1}+\mathrm{k}_{2}{ }^{*} \mathrm{SG}_{2}$

Eq. 6

$\mathrm{SG}_{1}$ é a tensão medida abaixo do furo de corrida

$\mathrm{SG}_{2}$ é a tensão medida próximo ao fundo do cadinho

$\mathrm{K}_{0}, \mathrm{k}_{1}$ e $\mathrm{k}_{2}$ são parâmetros determinados pela regressão linear.

Então apenas indicador do nível de líquidos foi encontrada:

Indicador do nível de líquidos= Pressão estimada* $\left(\mathrm{k}_{0}+\mathrm{k}_{1}{ }^{*} \mathrm{SG}_{1}+\mathrm{k}_{2}{ }^{*} \mathrm{SG}_{2}\right)$ - Pressão na zona de combustão

Eq. 7

O modelo demonstrou bons resultados mas às vezes este toma valores menores que zero.

Como observado, após algumas paradas do alto-forno apresenta valores baixos demonstrando que a validade desta equação em tais períodos deve ser aprimorada.

\section{CONCLUSÃO}

As condições do cadinho podem ser monitoradas de diversas maneiras. O problema consiste em obter métodos rápidos e ilustrativos. Um grande desafio é a estimativa do formato e tamanho do homem morto de maneira mais acurada.

\section{Agradecimentos}

Os autores agradecem a ABM a oportunidade da publicação deste trabalho.

\section{REFERÊNCIAS}

1 Saxén, H., Brännbacka, J., Pomeroy, D. Detection and quantification of the dead man floating state in the blast furnace. Metallurgical and Materials Transactions B, Volume 38B, June 2007-443

2 Brännbacka, J.,Saxén, H., Pomeroy,D. Identifying and quantifying a floating dead-man state in the blast furnace. AISTech 2005 Proceedings - Volume I p.173-182.

3 Torrkulla, J., Brannbacka, J., Saxén,H., Indicators of the state of the blast furnace hearth. Ironmaking conference proceedings, 2000. p481-487.

4 Peters, M., Rüther,P., Schmöle, P., Thiemann,T., Rödl, S. Determination of the liquid level in the hearth of the blast furnace. Ironmaking Conference Proceedings. p.283-295, 2001.

5 M.O. Hattink, S.A. Zaimi, H.-P. Ruther, O. Mielenz, H. Kochner, O. Havelange, D.Y. Sheng. Determination of factors influencing deadman position and evaluation of its impact on blast furnace lifetime. Research Programme of the Research Fund for Coal and Steel Coal RTD. Mid-term Report, 2007. 74 p.

6 Brännbacka, J.,Saxén, H., Pomeroy, D., Waller, M. Modelling the dead man floating behavior in a blast furnace. 3rd IAS Ironmaking Seminar, 2001, Buenos Aires, Argentina, pp.173-182. 